\title{
Ultra-thin Carbon Nanomechanical Resonator Fabrication and Its Dynamic Property Characterization
}

\author{
Reo Kometani, ${ }^{1,2^{*}}$ Mizue Sekine, ${ }^{2}$ and Shin'ichi Warisawa ${ }^{1}$ \\ ${ }^{1}$ Graduate School of Frontier Sciences, The University of Tokyo, \\ 5-1-5 Kashiwanoha, Kashiwa, Chiba 277-8563, Japan \\ ${ }^{2}$ Graduate School of Engineering, The University of Tokyo, 7-3-1 Hongo, Bunkyo, Tokyo 113-8656, Japan
}

(Received October 10, 2018; accepted January 11, 2019)

Keywords: single nanometer scale, nanomechanical resonator, nano-electromechanical systems (NEMS), diamond-like carbon (DLC), $Q$ factor, energy dispersion, focused ion beam (FIB)

The nanomechanical resonator is a useful structure to achieve various nanoelectromechanical systems (NEMS) sensing devices. In this study, systematic evaluations of the dynamic properties and mechanical material properties of an ultra-thin nanomechanical resonator made of diamond-like carbon (DLC) were carried out. As a result, the fabrication of an ultra-thin nanomechanial resonator with a thickness of 4.8-73 nm was achieved by focusedion-beam (FIB)-based nanofabrication. The energy dispersion of vibration depended on the surface area/volume $(S / V)$ ratio. A thinner nanomechanical resonator had higher sensitivity to pressure. Furthermore, we evaluated Young's modulus and density as mechanical material properties by measuring the resonant properties of the $\mathrm{DLC} / \mathrm{SiO}_{2}$ bilayer mechanical resonator. Young's modulus and density increased with decreasing thickness. This implied that the mechanical properties of nanoscale-thick DLC have a thickness dependence.

\section{Introduction}

The nanomechanical resonator is a key component of nano-electromechanical systems (NEMS) because it enables us to detect various small physical quantities using vibration. Thus far, various types of sensing using the nanomechanical resonator have been reported. Highly sensitive force sensing was achieved using a Si resonator ${ }^{(1,2)}$ and a carbon nanotube (CNT) resonator. ${ }^{(3)}$ Mass detection of yoctogram-order sensitivity using the CNT resonator was also demonstrated. ${ }^{(4)}$ Furthermore, displacement was detected with high sensitivity by integrating the resonator with a superconducting quantum interference device (SQUID) ${ }^{(5)}$ and a single-electron transistor (SET). ${ }^{(6)}$ In addition to these, the sensing of various targets was achieved, such as charge detection, ${ }^{(7)}$ light detection, ${ }^{(8,9)}$ and biomolecule detection. ${ }^{(10,11)}$ The nanomechanical resonator is a useful device for sensitive sensing.

In order to achieve high sensitivity, various studies on the resonant properties of nanomechanical resonators were carried out. The $Q$ factor is an important factor in achieving

*Corresponding author: e-mail: kometani@edu.k.u-tokyo.ac.jp

https://doi.org/10.18494/SAM.2019.2153 
high sensitivity. The $Q$ factor is defined by various energy dispersions ${ }^{(12)}$ such as air damping, support loss, thermoelastic damping, and surface loss. Its improvement was attempted by decreasing the energy dispersion through the application of $\operatorname{strain}^{(13-15)}$ and surface modification. ${ }^{(16,17)}$ Furthermore, the relationships among the resonant properties and resonator size were investigated to clarify the dynamics of nano- and microscale resonators. In these previous works, the relationships among the $Q$ factor, volume, ${ }^{(18)}$ thickness, ${ }^{(19)}$ and length ${ }^{(20)}$ were discussed for mechanical resonators made of various materials such as $\mathrm{Si}, \mathrm{CNT}$, and graphene. This previous research provided useful knowledge for achieving highly sensitive sensing using the nanomechanical resonator.

In this study, we carried out systematic evaluations of the dynamics of an ultimate-scale resonator to obtain knowledge about the single-nanoscale mechanical resonator. We focused on the thickness dependence of the dynamic properties of the nanomechanical resonator, and tried to achieve the precise evaluation by fabricating an ultra-thin mechanical resonator with various thicknesses on the same substrate to avoid process variation. Material mechanical properties such as Young's modulus and density were also evaluated.

\section{Fabrication of Ultra-thin Mechanical Resonator}

In this study, ultra-thin resonators were fabricated without an undercut structure for easy analysis of vibration properties, since the undercut structure affects the resonant characteristics. To realize a resonator without any undercut, the vibrating part and the supporting part of the resonator are integrated, and the supporting part is embedded in the Si substrate, as shown in Fig. 1(a). Also, to avoid the influence of stress on the resonant characteristics, a cantilever-type mechanical resonator was used in this experiment. This ultra-thin mechanical resonator was fabricated by a focused-ion-beam (FIB)-based nanofabrication technique.

Figure 2 shows a schematic of the fabrication process of the nanomechanial resonator. First, the Si substrate was etched with a $30 \mathrm{kV}$ Ga FIB to fabricate the trench for embedding the supporting parts of the resonator, as shown in Fig. 2(i). Then diamond-like carbon (DLC) was deposited as supporting parts in the trench structure of the Si substrate by FIB-induced chemical vapor deposition (FIB-CVD). Phenanthrene $\left(\mathrm{C}_{14} \mathrm{H}_{10}\right)$ was used as a gas source for

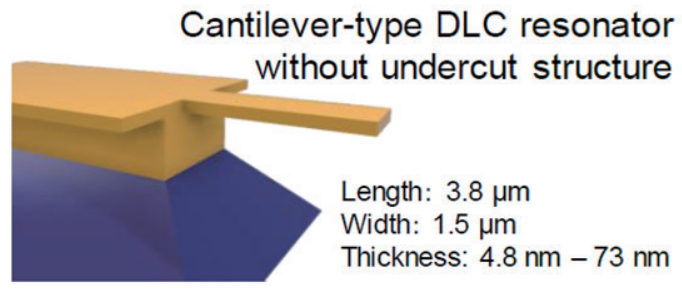

(a)

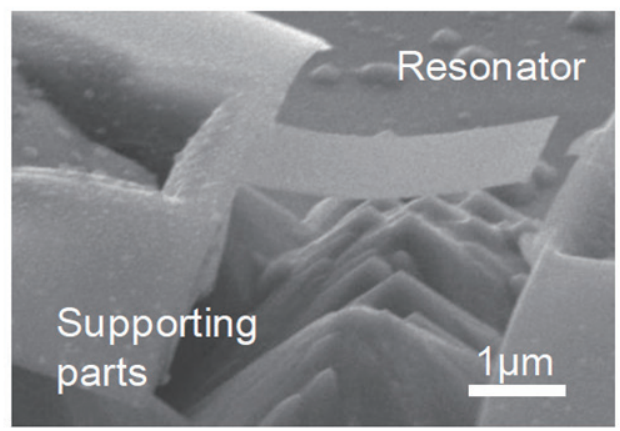

(b)

Fig. 1. (Color online) Cantilever-type DLC resonator without undercut structure. (a) Schematic of resonator structure. (b) SEM image of cantilever-type DLC resonator with a thickness of $4.8 \mathrm{~nm}$. 
(i)

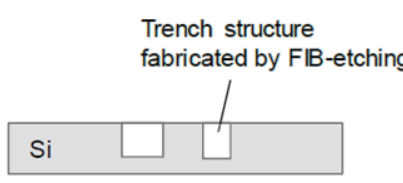

(iv)

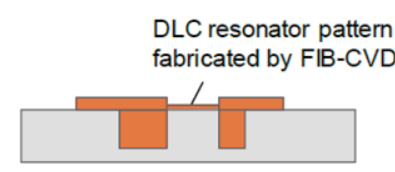

(ii) DLC supporting structure

Deposited by FIB-CVD

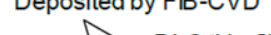

(iii)

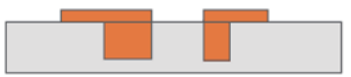

(v)

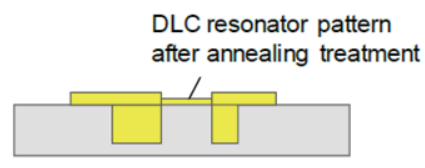

(vi)

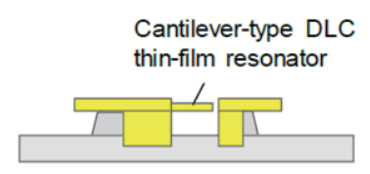

Fig. 2. (Color online) Schematic of fabrication of cantilever-type DLC resonator without undercut structure. (i) Trench structure fabrication by FIB etching. (ii) Fabrication of supporting parts by FIB-CVD. (iii) Surface cleaning of $\mathrm{Si}$ substrate by $\mathrm{O}_{2}$ RIE. (iv) DLC resonator pattern fabrication by FIB-CVD. (v) Ga removal by annealing. (vi) Cantilever-type DLC resonator fabrication by wet etching and FIB etching.

DLC deposition. ${ }^{(21,22)}$ The vacuum during deposition was approximately $5.0 \times 10^{-5} \mathrm{~Pa}$, and the ion dose was $50 \times 10^{15}$ ions $/ \mathrm{cm}^{2}$. Also, a DLC film was deposited as the top layer on the embedded DLC supporting parts, as shown in Fig. 2(ii). An ion dose of $800 \times 10^{15} \mathrm{ions} / \mathrm{cm}^{2}$ was used. Then, the Si substrate was cleaned by reactive ion etching using $\mathrm{O}_{2}$ gas to remove the DLC thin film deposited by the beam tail of the Ga ion beam during FIB-CVD, as shown in Fig. 2(iii). In this process, $\mathrm{RF}$ power, $\mathrm{O}_{2}$ pressure, and cleaning time were $100 \mathrm{~W}, 100 \mathrm{~Pa}$, and $270 \mathrm{~s}$, respectively. The DLC thin film, as the vibrating part, was deposited by FIB-CVD, as shown in Fig. 2(iv). FIB-CVD is a suitable technique for fabricating resonators with different film thicknesses on the same substrate because FIB-CVD is a bottom up nanofabrication technique. Also, FIB-CVD enables the precise control of film thickness. Ion doses of $3.0 \times 10^{15}$ to $50 \times 10^{15}$ ions $/ \mathrm{cm}^{2}$ were used for ultra-thin-film deposition. Then, the DLC thin film and Si substrate were annealed at $800{ }^{\circ} \mathrm{C}$ for $1 \mathrm{~h}$ under a vacuum of $1.0 \times 10^{-2} \mathrm{~Pa}$ to remove the Ga implanted by FIB, as shown in Fig. 2(v). This annealing treatment was necessary as a Si layer remains under the DLC layer in the next wet etching unless Ga is removed. The Si substrate was etched by wet etching to fabricate the doubly clamped resonant structure. In this process, tetramethylammonium hydroxide (TMAH, Pure Etch 160, Hayashi Pure Chemical Ind.) at 68 ${ }^{\circ} \mathrm{C}$ was used. Finally, a cantilever-type resonant structure was obtained by etching one clamp of the doubly clamped resonant structure using a $30 \mathrm{kV}$ FIB, as shown in Fig. 2(vi). In this fabrication, in order to avoid the stiction of the resonant structure by wet etching, the cantilevertype structure was fabricated after the fabrication of the doubly clamped structure.

In this study, the fabrication of ultra-thin-film DLC mechanical resonators with thicknesses of 4.8-73 nm was achieved. The thickness of the DLC resonator was obtained by measuring the thickness of the DLC resonator pattern by atomic force microscopy (AFM) before wet etching. Length and width were 3.8 and $1.5 \mu \mathrm{m}$, respectively. Figure 1(b) shows a scanning electron microscopy (SEM) image of an ultra-thin-film DLC resonator with a thickness of $4.8 \mathrm{~nm}$. 


\section{Evaluation of Resonant Properties}

Resonant properties were measured using an optical heterodyne vibrometer. Vibration was excited by photothermal excitation using a semiconductor laser with a wavelength of $408 \mathrm{~nm}$. The vibration spectrum was measured using a He-Ne laser at room temperature.

Figures 3(a) and 3(b) show the resonant characteristics of the cantilever-type DLC nanomechanical resonator. In this measurement, the resonant frequency of the 1st bending mode of the resonator was measured under a vacuum of $2.2 \times 10^{-3} \mathrm{~Pa}$. The resonant frequency depended on the thickness of the nanomechanical resonator and changed almost linearly with film thickness. Also, Fig. 3(b) shows the surface area/volume $(S / V)$ ratio dependence of energy dispersion $(1 / Q)$. $1 / Q$ increased with an increase in the $S / V$ ratio. It seems that this dependence was caused by the surface loss of vibration. This shows good agreement with the previous research. $^{(18,19)}$

Furthermore, the pressure dependence of $1 / Q$ was evaluated in this study. In this experiment, the chamber pressure was changed from $2.2 \times 10^{-3}$ to $1.4 \times 10^{4} \mathrm{~Pa}$. Figure 4 shows the relationship between the chamber pressure, $Q$ factor, and $1 / Q$. As shown in the inset graph in Fig. 4, the vacuum under which the $Q$ value starts to improve was higher with a thinner resonator. The $1 / Q$ of a thinner resonator was larger under lower vacuum. This indicates that a thinner mechanical resonator has higher sensitivity to the chamber pressure and surrounding environment, as we expected. This result also agrees well with the previous research. ${ }^{(18)}$ The $Q$ factor of the nanomechanical resonator with a single-digit-nanometer-order thickness was not saturated even under a vacuum of $10^{-1} \mathrm{~Pa}$. Because its environment becomes a molecular region as the vacuum pressure decreases, this result implies that ultra-thin-film resonators are even sensitive to the effects of the collision of residual air molecules, for example.

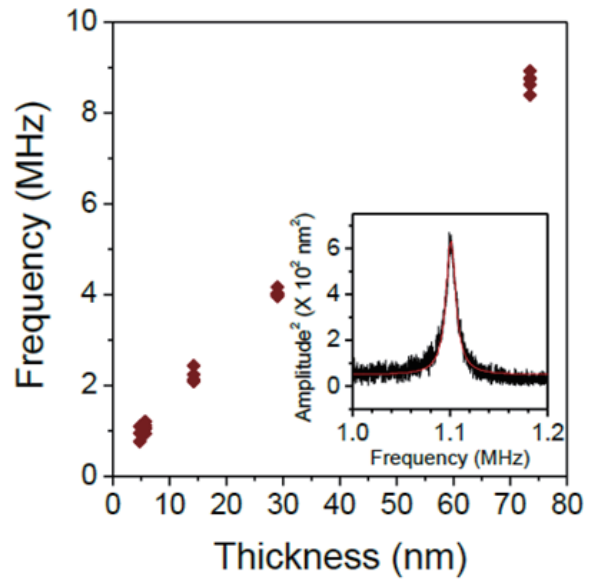

(a)

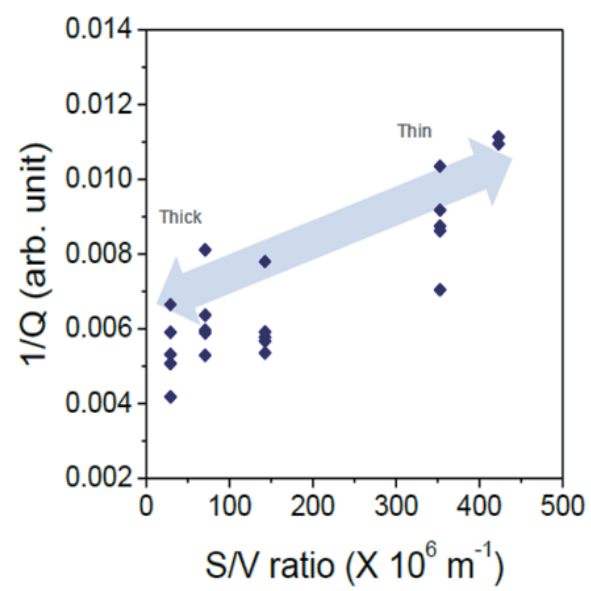

(b)

Fig. 3. (Color online) Resonant properties of cantilever-type DLC resonator. (a) Relationship between thickness and resonant frequency. Inset shows the vibration spectrum of the DLC resonator with a thickness of $4.8 \mathrm{~nm}$. (b) Surface area/volume $(S / V)$ ratio dependence of energy dispersion $(1 / Q)$. 


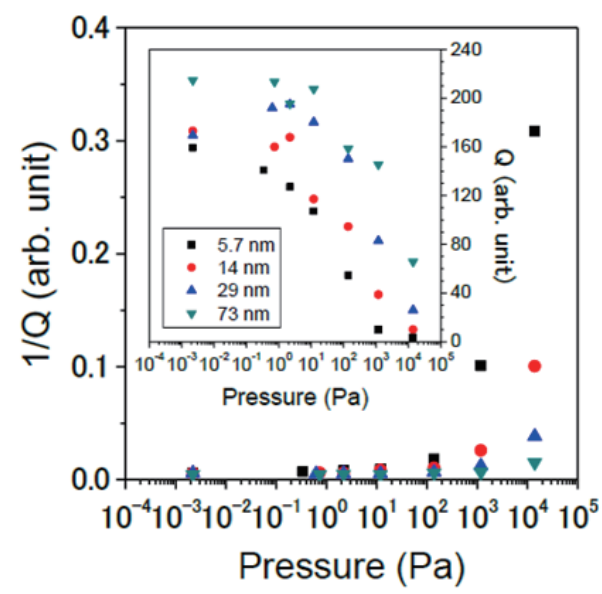

Fig. 4. (Color online) Pressure dependence of energy dispersion $(1 / Q)$. Inset shows the relationship between pressure and $Q$ factor.

\section{Material Mechanical Properties Evaluation}

Material mechanical properties were also evaluated to clarify the dynamics of the ultrathin-film mechanical resonator. We examined the resonant properties of the DLC mechanical resonator and the DLC/SiO 2 bilayer resonator to obtain Young's modulus and density. The Young's modulus/density $\left(E_{c} / \rho_{c}\right)$ ratio was calculated using the resonant properties of the DLC mechanical resonator shown in Fig. 3(a). In addition to this, the resonant frequency ratios $\left(f_{b i} / f_{s}\right)$ of the $\mathrm{DLC} / \mathrm{SiO}_{2}$ bilayer resonator and $\mathrm{SiO}_{2}$ resonator were obtained in order to calculate Young's modulus and density. $f_{b i}$ and $f_{s}$ are resonant frequencies of the $\mathrm{DLC} / \mathrm{SiO}_{2}$ bilayer resonator and $\mathrm{SiO}_{2}$ resonator, respectively.

Figure 5(a) shows a schematic of the $\mathrm{DLC} / \mathrm{SiO}_{2}$ bilayer resonator. Figure 5(b) shows a SEM image of the $\mathrm{DLC} / \mathrm{SiO}_{2}$ bilayer resonator fabricated by FIB etching, wet etching, FIB-CVD, and annealing. First, in the fabrication of the $\mathrm{DLC} / \mathrm{SiO}_{2}$ bilayer resonator, the shape of the resonator was patterned on a $\mathrm{SiO}_{2} / \mathrm{Si}$ substrate by etching using a $30 \mathrm{kV}$ Ga FIB with a beam current of $738 \mathrm{pA}$. The thickness of the $\mathrm{SiO}_{2}$ layer was $280 \mathrm{~nm}$. Then, because the incorporated $\mathrm{Ga}$ inhibited the wet etching of $\mathrm{Si}, 800{ }^{\circ} \mathrm{C}$ annealing for $1 \mathrm{~h}$ was carried out under a vacuum of $10^{-2} \mathrm{~Pa}$ in order to remove the incorporated $\mathrm{Ga}$. The wet etching was carried out using $68^{\circ} \mathrm{C}$ TMAH (Pure Etch 160, Hayashi Pure Chemical Ind.) to fabricate the doubly clamped structure made of $\mathrm{SiO}_{2}$. After the wet etching, the DLC thin film was deposited by FIB-CVD. A $30 \mathrm{kV}$ Ga FIB with a beam current of $13 \mathrm{pA}$ was used. Ion doses of $2.0 \times 10^{15}$ to $50 \times 10^{15} \mathrm{ions} / \mathrm{cm}^{2}$ were used. After the DLC thin-film deposition on the $\mathrm{SiO}_{2}$ doubly clamped structure, the DLC thin film was annealed at $800^{\circ} \mathrm{C}$ for $1 \mathrm{~h}$ under a vacuum of $10^{-2} \mathrm{~Pa}$ to remove the incorporated Ga. Finally, the cantilever-type $\mathrm{DLC} / \mathrm{SiO}_{2}$ bilayer resonator was fabricated by etching one clamp of the doubly clamped structure using an FIB with a beam current of $85 \mathrm{pA}$. Also, both sides of the clamp of the cantilever-type resonator were etched with an FIB to eliminate the influence of the undercut structure on the resonant properties. By these fabrication processes, the $\mathrm{DLC} / \mathrm{SiO}_{2}$ bilayer resonator with a length and width of 8 and $1.6 \mu \mathrm{m}$, respectively, was 


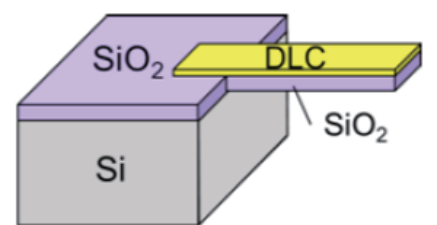

(a)

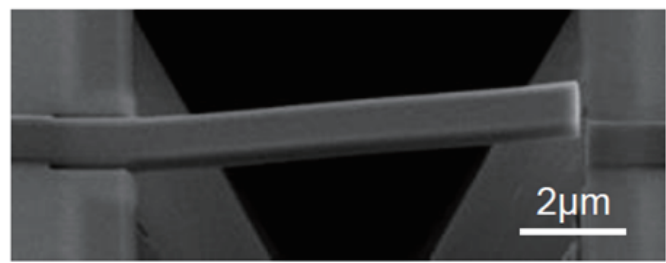

(b)

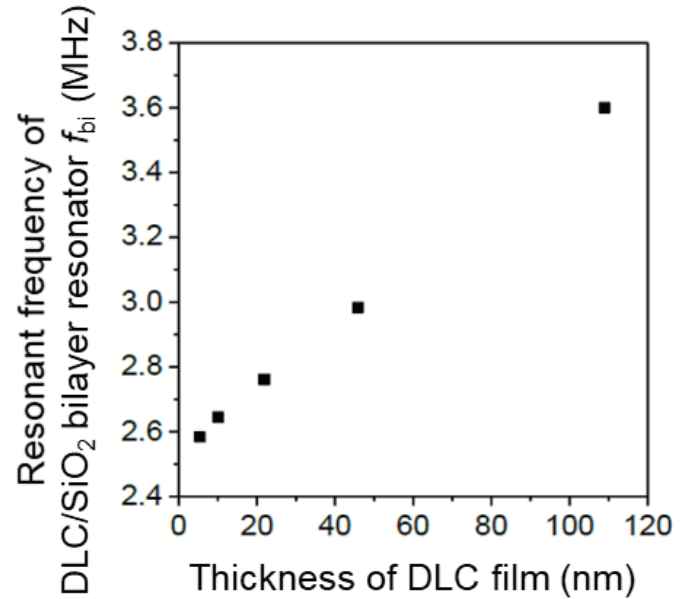

(c)

Fig. 5. (Color online) $\mathrm{DLC} / \mathrm{SiO}_{2}$ bilayer resonator used in the mechanical material properties evaluation. (a) Schematic of $\mathrm{DLC} / \mathrm{SiO}_{2}$ bilayer resonator. (b) $\mathrm{SEM}$ image of a $\mathrm{DLC} / \mathrm{SiO}_{2}$ bilayer resonator. (c) Resonant frequency of $\mathrm{DLC} / \mathrm{SiO}_{2}$ bilayer resonators.

obtained. The thicknesses of the DLC thin film were 5.5, 10.1, 21.9, 45.9, and $109 \mathrm{~nm}$. The $\mathrm{SiO}_{2}$ resonator was also fabricated by the same process except the process of DLC film deposition.

In the calculation of Young's modulus and density, a model ${ }^{(23)}$ of the bilayer beam was used to obtain $f_{b i} / f_{s}$, which is defined as

$$
\left(\frac{f_{b i}}{f_{s}}\right)^{2}=\frac{\left(E_{r} t_{r}^{3}+1\right)\left(E_{r} t_{r}+1\right)+3 E_{r} t_{r}\left(t_{r}+1\right)^{2}}{\left(\rho_{r} t_{r}+1\right)\left(E_{r} t_{r}+1\right)},
$$

where $E_{r}=E_{c} / E_{s}, \rho_{r}=\rho_{c} / \rho_{s}$, and $t_{r}=t_{c} / t_{s} . E_{c}, \rho_{c}$, and $t_{c}$ are Young's modulus, density, and thickness of the LC film, respectively. $E_{S}, \rho_{s}$, and $t_{s}$ were $74 \mathrm{GPa},{ }^{(24)} 2200 \mathrm{~kg} / \mathrm{m}^{3}$, ${ }^{(24)}$ and $280 \mathrm{~nm}$ as Young's modulus, density, and thickness of the $\mathrm{SiO}_{2}$ resonator, respectively. The relationship between $E_{c}, \rho_{c}$, and $f_{c}$ is

$$
\rho_{c}=k E_{c}, k=0.0261 \frac{1}{l_{c}^{4}}\left(\frac{t_{c}}{f_{c}}\right)^{2}
$$

$f_{c}$ and $l_{c}$ are the resonant frequency and the length of the DLC thin-film resonator, respectively. By using these relationships, the following equation is derived.

$$
\left(\frac{t_{r}^{4}}{E_{s}^{2}}-\frac{f_{b i}^{2} t_{r}^{2} k}{f_{s}^{2} E_{s} \rho_{s}}\right) E_{c}^{2}+\frac{t_{r}}{E_{s}}\left[4 t_{r}^{2}+6 t_{r}+4-\frac{f_{b i}^{2}}{f_{s}^{2}}\left(\frac{E_{s} k}{\rho_{s}}+1\right)\right] E_{c}+\left(1-\frac{f_{b i}^{2}}{f_{s}^{2}}\right)=0
$$

$E_{c}$ was derived from Eq. (3). $f_{b i}$ and $f_{s}$ were obtained by vibration measurements. Finally, $\rho_{c}$ was obtained using the $E_{c} / \rho_{c}$ ratio. 
Figure 6 shows the relationships between thickness, Young's modulus, and density. A thinner DLC has a larger Young's modulus and a higher density, as shown in Fig. 6. There are several reports about the size dependence of Young's modulus of nanoscale Si structures. ${ }^{(25,26)}$ In those previous reports, it was discussed that the causes of the size dependence are surface relaxation and surface stress. There is a possibility that the increase in Young's modulus was caused by the surface stress although additional precise evaluations are needed to clarify the material properties. Moreover, this surface stress and surface condition cause the bending of the cantilever, as shown in Fig. 2(b). Also, although the mechanism behind the thickness dependence of density is unclear, there is a possibility that the influence of substances adsorbed on the DLC surface, such as residual gas molecules, may appear in the evaluation of density. In order to clarify this, it is necessary to perform surface analysis in detail. Furthermore, the density was lower than those of typical carbon materials such as diamond, graphite, and DLC. DLC deposited by FIB-CVD has a density of approximately $1.9 \mathrm{~g} / \mathrm{cm}^{3}$. (27) This density is for DLC after it was annealed at $600{ }^{\circ} \mathrm{C}$. The low density seems to be caused by the removal of Ga upon annealing treatment, because the structural defect developed after annealing treatment. ${ }^{(28)}$ In addition, the deposition rate was higher on the $\mathrm{SiO}_{2}$ substrate than on the $\mathrm{Si}$ substrate. For example, the deposition rate was 1.49 times higher with the dose of $50 \times 10^{15}$ ions $/ \mathrm{cm}^{2}$. Although it is considered that it does not have a large influence, there is a possibility that the difference in the deposition rate affects many material properties. This may also give rise to errors to the evaluation of material properties.

Although it is mentioned that the increase in $1 / Q$ was caused by the surface loss of vibration in the resonant property evaluation, the film thickness dependences of Young's modulus and density suggest that volume loss is also a factor affecting $1 / Q$. However, in order to consider the influence of the volume loss on the resonance characteristic, various additional experiments are necessary, such as the evaluation of the resonant properties of the nanomechanical resonator with the same size but different Young's modulus. At the least, the film thickness dependence of material properties implies the importance of considering volume loss on the resonant property of the ultra-thin-film resonator.

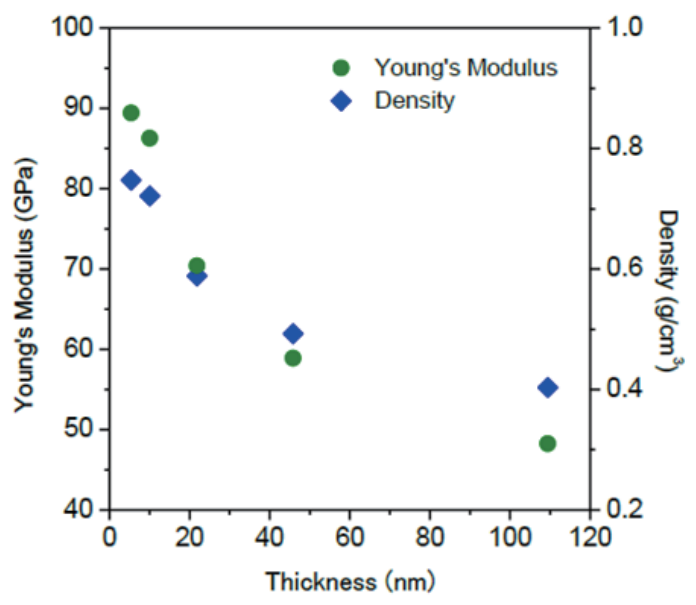

Fig. 6. (Color online) Relationship of thickness with Young's modulus and density. 


\section{Conclusions}

The fabrication of an ultra-thin-film DLC nanomechanical resonator was achieved using FIB technologies, and its dynamic properties and mechanical material properties were evaluated. As a result, $1 / Q$ was found to depend on the $S / V$ ratio and pressure, as found in the previous works. The ultra-thin-film resonator with a thickness of single-digit nanometer order had a larger $1 / Q$ owing to surface loss and air damping. Furthermore, Young's modulus and density were measured in this study. Young's modulus and density increased with a decrease in the thickness of DLC. Influences such as the surface effect may be the key in clarifying this size dependence. The dynamic properties and material properties obtained in this study will be useful in achieving higher performance and higher reliability of nanoscale sensing devices.

\section{Acknowledgments}

This work was supported by JSPS KAKENHI Grant Number 17H03198.

\section{References}

1 H. J. Mamin and D. Rugar: Appl. Phys. Lett. 79 (2001) 3358. https://doi.org/10.1063/1.1418256

2 T. D. Stowe, K. Yasumura, T. W. Kenny, D. Botkin, K. Wago, and D. Rugar: Appl. Phys. Lett. 71 (1997) 288. https://doi.org/10.1063/1.119522

3 J. Moser, J. Güttinger, A. Eichler, M. J. Esplandiu, D. E. Liu, M. I. Dykman, and A. Bachtold: Nat. Nanotechnol. 8 (2013) 493. https://doi.org/10.1038/nnano.2013.97

4 J. Chaste, A. Eichler, J. Moser, G. Ceballos, R. Rurali, and A. Bachtold: Nat. Nanotechnol. 7 (2012) 301. https://doi.org/10.1038/nnano.2012.42

5 S. Etaki, M. Poot, I. Mahboob, K. Onomitsu, H. Yamaguchi, and H. S. J. van der Zant: Nat. Phys. 4 (2008) 785. https://doi.org/10.1038/nphys1057

6 R. G. Knobel and A. N. Cleland: Nature 424 (2003) 291. https://doi.org/10.1038/nature01773

7 A. N. Cleland and M. L. Roukes: Nature 392 (1997) 160. https://doi.org/10.1038/32373

8 T. Ono and M. Esashi: Rev. Sci. Instrum. 74 (2003) 5141. https://doi.org/10.1063/1.1623627

9 E. Maeda and R. Kometani: Appl. Phys. Lett. 111 (2017) 013102-1-4. https://doi.org/10.1063/1.4991683

10 M. Varshney, P. S. Waggoner, C. P. Tan, K. Aubin, R. A. Montagna, and H. G. Craighead: Anal. Chem. 80 (2008) 2141. https://doi.org/10.1021/ac702153p

11 B. Ilic, Y. Yang, K. Aubin, R. Reichenbach, S. Krylov, and H. G. Craighead: Nano Lett. 5 (2005) 925. https:// doi.org/10.1021/n1050456k

12 K. Naeli and O. Brand: J. Appl. Phys. 105 (2009) 014908. https://doi.org/10.1063/1.3062204

13 S. S. Verbridge, D. F. Shapiro, H. G. Craighead, and J. M. Parpia: Nano Lett. 7 (2007) 1728.

14 H. Yamaguchi, K. Kato, Y. Nakai, K. Onomitsu, S. Warisawa, and S. Ishihara: Appl. Phys. Lett. 92 (2008) 251913. https://doi.org/10.1063/1.2952957

15 Y. Oshidari, T. Hatakeyama, R. Kometanil, S. Warisawa, and S. Ishihara: Appl. Phys. Express 5 (2012) 117201. http://dx.doi.org/10.1143/APEX.5.117201

16 Y. Wang, J. A. Henry, A. T. Zehnder, and M. A. Hines: J. Phys. Chem. B 107 (2003) 14270. https://doi. org/1021/jp0360164

17 H. Shimizu, J.-J. Delaunay, R. Kometani, S. Warisawa, and S. Ishihara: Jpn. J. Appl. Phys. 49 (2010) 06GN13. https://doi.org/10.1143/JJAP.49.06GN13

18 M. Imboden and P. Mohanty: Phys. Rep. 534 (2014) 89. https://doi.org/10.1016/j.physrep.2013.09.003

19 K. Y. Yasumura, T. D. Stowe, E. M. Chow, T. Pfafman, T. W. Kenny, B. C. Stipe, and D. Rugar: J. Microelectromech. Syst. 9 (2000) 117. https://doi.org/10.1109/84.825786

20 K. Naeli and O. Brand: J. Appl. Phys. 105 (2009) 014908-1-10. http://dx.doi.org/10.1063/1.3062204

21 K. Kanda, J. Igaki, Y. Kato, R. Kometani, A. Saikubo, and S. Matsui: Radiat. Phys. Chem. 75 (2006) 1850. https://doi.org/10.1016/j.radphyschem.2005.07.039 
22 R. Kometani, T. Ichihachi, K. Kanda, T. Suzuki, K, Niihara, S. Ishihara, T. Kaito, and S. Matsui: J. Vac. Sci. Technol., B 26 (2008) 2628. https://doi.org/10.1116/1.2978400

23 R. Whiting, M. A. Angadi, and S. Tripathi: Mater. Sci. Eng., B 30 (1995) 35. https://doi.org/10.1016/09215107(94)01133-8

24 R. J. Hussey and J. Wilson: Advanced Technical Ceramics Directory and Databook, Technology \& Engineering (Springer, 2012). ISBN: 1441986626

25 M. Ishida, J. Fujita, and Y. Ochiai: J. Vac. Sci. Technol., B 20 (2002) 2784. https://doi.org/10.1116/1.1526699

26 X. Li, T. Ono, Y. Wang, and M. Esashi: Appl. Phys. Lett. 83 (2003) 3081. https://doi.org/10.1063/1.1618369

27 H. Yu, C. Sun, W. W. Zhang, S. Y. Lei, and Q. A. Huang: J. Nanomater. 2013 (2013) 319302-1-5. http://dx.doi. org/10.1155/2013/319302

28 R. Kometani, K. Yusa, S. Warisawa, and S. Ishihara: J. Vac. Sci. Technol., B 28 (2010) C6F38. https://doi. org/10.1116/1.3504584 